\title{
q-enumeration of words by their total variation $\|^{i}$
}

\author{
Ligia L. Cristea $\|^{\text {I }}$ and Helmut Prodinger非 \\ ${ }^{1}$ Technische Universität Graz, Institut für Mathematik, Graz, Austria. \\ ${ }^{2}$ Mathematics Department, Stellenbosch University, Stellenbosch, South Africa.
}

received $9^{\text {th }}$ December 2009, revised 31 ${ }^{\text {st }}$ July 2010, accepted 31 ${ }^{\text {st }}$ July 2010.

In recent work, Mansour [Discrete Math. Theoret. Computer Science 11, 2009, 173-186] considers the problem of enumerating all words of length $n$ over $\{1,2, \ldots, k\}$ (where $k$ is a given integer), that have the total variation equal to a given integer $m$. In the present paper we study various types of random words over the infinite alphabet $\mathbb{N}$, where the letters have geometric probabilities. We are interested in the probabilities of words of given type to have a given total variation.

Keywords: words, $q$-enumeration, generating functions, up-down words, Carlitz words

\section{Introduction}

In recent work Mansour [6] considers the problem of enumerating all words of length $n$ over $\{1,2, \ldots, k\}$ (where $k$ is a given integer), that have the total variation equal to a given integer $m$, where the total variation of a word is defined as the sum of the absolute values of the differences of adjacent letters of the word (as in formula (1)). Previously, several authors have studied the statistic "total variation" in various contexts. For more information we refer to Mansour [6].

In the present paper we consider words over the infinite alphabet $\mathbb{N}$. We assume that for any word $\omega=\omega_{1} \omega_{2} \ldots \omega_{n}$ the occurrence probability of the letter $j \in \mathbb{N}$ is $\mathbb{P}\left(\omega_{i}=j\right)=p q^{j-1}$, independently, for all $n, i, j \in \mathbb{N}$ with $i \leq n$, where $0<p, q<1$ and $p+q=1$ and investigate the probability of a word of given length to have the total variation $u(\omega)=r$. We consider three different families of words over $\mathbb{N}$.

In Section 2 we study the most general case, namely words over $\mathbb{N}$ (with no other restrictions on the occurrences of letters within the words). In Section 3 we consider up-down words over $\mathbb{N}$ that follow the pattern $<\geq<\geq \cdots<\geq$. We first study words of odd length and then use the result for the study of words of even length. The last section deals with Carlitz words over $\mathbb{N}$, i.e., words with the property that any two adjacent letters are distinct.

\footnotetext{
$\dagger$ Parts of this work were done during a research stay of the of the first author at the Mathematics Department of the University of Stellenbosch.

¥This author is supported by the Austrian Research Foundation (FWF), Project P20412-N18. Email: strublistea@gmail. com

$\S$ This author is supported by the South African Science Foundation NRF, Grant 2053748. Email: hproding@ sun . ac . za 
For all studied families of words we use the method of "adding a slice": we start with a word of length $n$ with total variation $r$ and last letter $i \in \mathbb{N}$, then add one letter (except for the case of up-down words of odd length where we add two letters) and see what influence this has on the total variation of the word. We use generating functions in order to obtain the probabilities that we are looking for. The method that we use can be applied to further families of words over $\mathbb{N}$.

\section{The general case: words over $\mathbb{N}$}

Let $\mathcal{W}_{n}$ denote the set of words of length $n$ over $\mathbb{N}$, for $n \geq 1$, and $\mathcal{W}^{+}=\bigcup_{n>1} \mathcal{W}_{n}$ is the set of all finite words over $\mathbb{N}$. For $\omega \in \mathcal{W}_{n}, n \geq 1, \omega=\omega_{1} \omega_{2} \ldots \omega_{n}$, the total variation of the word $\omega$ is defined (see Mansour [6]) as

$$
u(\omega)=\sum_{i=1}^{n-1}\left|\omega_{i+1}-\omega_{i}\right| .
$$

We are interested in solving the following problem.

Problem: For given $n \geq 1$ and $r \geq 0$, what is the probability of a word $\omega \in \mathcal{W}_{n}$ to have total variation $u(\omega)=r ?$

Therefore we introduce the following generating functions. Let

$$
f(z, x)=\sum_{n \geq 1, r \geq 0} p(n, r) z^{n} x^{r},
$$

where $p(n, r)$ is the probability that a word of length $n$ has total variation $r$. Moreover, let

$$
F(z, x, t)=\sum_{n \geq 1, r \geq 0, i \geq 1} g(n, r, i) z^{n} x^{r} t^{i},
$$

where $g(n, r, i)$ is the occurrence probability of a word of length $n$ with last letter $i\left(\omega_{n}=i\right)$ and total variation $r$. We have $f(z, x)=F(z, x, 1)$.

In order to find the occurrence probabilities of words with $n$ letters and total variation $r$, we proceed inductively as follows.

Assume we have a random word of length $n$ with last letter $i, \omega=\omega_{1} \ldots \omega_{n-1} i$, which has total variation $r$, with arbitrarily fixed $i \geq 1$ and $r \geq 0$. Suppose we add a letter, $j$, after the last letter $i$, and thus get a new word of length $n+1, \omega^{\prime}=\omega_{1} \ldots \omega_{n-1} i j$. Then either $j>i$ and the new word $\omega^{\prime}$ has total variation $r+j-i$, or $j \leq i$ and then $\omega^{\prime}$ has total variation $r+i-j$.

This is reflected by the following expression

$$
\sum_{j=1}^{i} p q^{j-1} \cdot x^{i-j} \cdot t^{j}+\sum_{j \geq i+1} p q^{j-1} \cdot x^{j-i} \cdot t^{j} .
$$

This leads to the substitution

$$
t^{i} \longrightarrow \frac{p t}{x-q t} x^{i}+\frac{p t T}{x-q t}(q t)^{i}
$$


with $T=T(x, t)=\frac{x^{2}-1}{1-x q t}$.

Our aim is to find the probabilities $p(n, r)$, for $n \geq 1$ and $r \geq 0$. Therefore we compute the generating function $f(z, x)$.

Let $a_{n}(x, t), n \geq 1$, be the functions with the property

$$
F(z, x, t)=\sum_{n \geq 1} a_{n}(x, t) z^{n} .
$$

For given $n \geq 1$, we obtain from (5) that

$$
a_{n+1}(x, t)=\frac{p t}{1-q t} a_{n}(x, x)+\frac{p t}{x-q t} T(x, t) a_{n}(x, q t),
$$

which, after multiplication by $z^{n+1}$ and then summing up for $n \geq 1$, leads to

$$
F(z, x, t)=a_{1}(x, t) z+z \frac{p t}{1-q t} F(z, x, x)+z \frac{p t}{x-q t} T(x, t) F(z, x, q t),
$$

where $a_{1}(x, t)=\sum_{k \geq 1} p q^{k-1} t^{k}=\frac{p t}{1-q t}$. This can now be solved by iteration:

$$
\begin{aligned}
F(z, x, t)= & \frac{p t}{1-q t} z+\frac{p t}{x-q t} z F(z, x, x)+\frac{p t}{x-q t} z T(x, t)[ \\
& \frac{p q t}{1-q^{2} t} z+\frac{p q t}{x-q^{2} t} z F(z, x, x)+\frac{p q t}{x-q^{2} t} z T(x, q t)[ \\
& \frac{p q^{2} t}{1-q^{3} t} z+\frac{p q^{2} t}{x-q^{3} t} z F(z, x, x)+\frac{p q^{2} t}{x-q^{3} t} z T\left(x, q^{2} t\right)[ \\
& \frac{p q^{3} t}{1-q^{4} t} z+\frac{p q^{3} t}{x-q^{4} t} z F(z, x, x)+\frac{p q^{3} t}{x-q^{4} t} z T\left(x, q^{3} t\right)[ \\
& \left.\left.\left.\left.\frac{p q^{4} t}{1-q^{5} t} z+\ldots\right]\right]\right]\right] .
\end{aligned}
$$

Herefrom we obtain

$$
F(z, x, t)=\sum_{k \geq 0} p^{k+1} t^{k+1} z^{k+1} q^{\frac{k(k+1)}{2}} \prod_{l=1}^{k} \frac{T\left(x, q^{l-1} t\right)}{x-q^{l} t}\left(\frac{1}{1-q^{k+1} t}+\frac{1}{x-q^{k+1} t} F(z, x, x)\right) .
$$

For $t=x$, we get

$$
F(z, x, x)=\sum_{k \geq 0} p^{k+1} x^{k+1} z^{k+1} q^{\frac{k(k+1)}{2}} \prod_{l=1}^{k} \frac{T\left(x, q^{l-1} x\right)}{x-q^{l} x}\left(\frac{1}{1-q^{k+1} x}+\frac{1}{x-q^{k+1} x} F(z, x, x)\right),
$$

and thus

$$
F(z, x, x)=\frac{\sum_{k \geq 0} p^{k+1} x^{k+1} z^{k+1} q^{\frac{k(k+1)}{2}} \frac{1}{1-q^{k+1} x} \prod_{l=1}^{k} \frac{T\left(x, q^{l-1} x\right)}{x-q^{l} x}}{1-\sum_{k \geq 0} p^{k+1} x^{k} z^{k+1} q^{\frac{k(k+1)}{2}} \frac{1}{1-q^{k+1}} \prod_{l=1}^{k} \frac{T\left(x, q^{l-1} x\right)}{x-q^{l} x}}
$$


We obtain

$$
\begin{aligned}
F(z, x, t)= & \sum_{n \geq 0} p^{n+1} t^{n+1} z^{n+1} q^{\frac{n(n+1)}{2}}\left(\frac{1}{1-q^{n+1} t}\right. \\
& \left.+\frac{1}{x-q^{n+1} t} \frac{\sum_{k \geq 0} p^{k+1} x^{k+1} z^{k+1} q^{\frac{k(k+1)}{2}} \frac{1}{1-q^{k+1} x} \prod_{l=1}^{k} \frac{T\left(x, q^{l-1} x\right)}{x-q^{l} x}}{1-\sum_{k \geq 0} p^{k+1} x^{k} z^{k+1} q^{\frac{k(k+1}{2}} \frac{1}{1-q^{k+1}} \prod_{l=1}^{k} \frac{T\left(x, q^{l-1} x\right)}{x-q^{l} x}}\right) \cdot \prod_{l=1}^{n} \frac{T\left(x, q^{l-1} t\right)}{x-q^{l} t} .
\end{aligned}
$$

In the following we use the notation $(x ; q)_{n}:=\prod_{j=0}^{n-1}\left(1-x q^{j}\right)$ for $n \geq 1$, and $(x ; q)_{0}=1$.

Since

$$
\prod_{l=1}^{n} T\left(x, q^{l-1} t\right)=\frac{\left(x^{2}-1\right)^{n}}{(q x t ; q)_{n}}
$$

we have

$$
\begin{aligned}
F(z, x, t)= & \sum_{n \geq 0} p^{n+1} t^{n+1} z^{n+1} q^{\frac{n(n+1)}{2}}\left(\frac{1}{1-q^{n+1} t}\right. \\
& \left.+\frac{1}{x-q^{n+1} t} \cdot \frac{\sum_{k \geq 0} p^{k+1} x^{k+1} z^{k+1} q^{\frac{k(k+1)}{2}} \cdot \frac{1}{1-q^{k+1} x} \cdot \frac{\left(x^{2}-1\right)^{k}}{x^{k}\left(q x^{2} ; q\right)_{k}(q ; q)_{k}}}{1-\sum_{k \geq 0} p^{k+1} x^{k} z^{k+1} q^{\frac{k(k+1)}{2}} \cdot \frac{1}{1-q^{k+1}} \cdot \frac{\left(x^{2}-1\right)^{k}}{x^{k}\left(q x^{2} ; q\right)_{k}(q ; q)_{k}}}\right) \frac{\left(x^{2}-1\right)^{n}}{x^{n}\left(q \frac{t}{x}\right)_{n}(x q t)_{n}} \\
= & \sum_{n \geq 0} p^{n+1} t^{n+1} z^{n+1} q^{\frac{n(n+1)}{2}}\left(\frac{1}{1-q^{n+1} t}\right. \\
& \left.+\frac{1}{x-q^{n+1} t} \cdot \frac{\sum_{k \geq 0} p^{k+1} x z^{k+1} q^{\frac{k(k+1)}{2}} \cdot \frac{1}{1-q^{k+1} x} \cdot \frac{\left(x^{2}-1\right)^{k}}{\left(q x^{2} ; q\right)_{k}(q ; q)_{k}}}{1-\sum_{k \geq 0} p^{k+1} z^{k+1} q^{\frac{k(k+1)}{2}} \cdot \frac{1}{1-q^{k+1}} \cdot \frac{\left(x^{2}-1\right)^{k}}{\left(q x^{2} ; q\right)_{k}(q ; q)_{k}}}\right) \frac{\left(x^{2}-1\right)^{n}}{x^{n}\left(q \frac{t}{x} ; q\right)_{n}(x q t ; q)_{n}} .
\end{aligned}
$$

For $t=1$ we obtain

$$
\begin{aligned}
f(z, x)= & F(z, x, 1) \\
= & \sum_{n \geq 0} p^{n+1} z^{n+1} q^{\frac{n(n+1)}{2}}\left(\frac{1}{1-q^{n+1}}\right. \\
& \left.+\frac{1}{x-q^{n+1}} \cdot \frac{\sum_{k \geq 0} p^{k+1} x z^{k+1} q^{\frac{k(k+1)}{2}} \cdot \frac{1}{1-q^{k+1} x} \cdot \frac{\left(x^{2}-1\right)^{k}}{\left(q x^{2} ; q\right) k(q ; q)_{k}}}{1-\sum_{k \geq 0} p^{k+1} z^{k+1} q^{\frac{k(k+1)}{2}} \cdot \frac{1}{1-q^{k+1}} \cdot \frac{\left(x^{2}-1\right)^{k}}{\left(q x^{2} ; q\right)_{k}(q ; q)_{k}}}\right) \frac{\left(x^{2}-1\right)^{n}}{x^{n}\left(\frac{q}{x} ; q\right)_{n}(x q ; q)_{n}} .
\end{aligned}
$$

For $x=1$ we obtain

$$
f(z, 1)=F(z, 1,1)=\frac{z}{1-z},
$$

which corresponds to the case when neither the last letter nor the total variation of the words are considered. 
Furthermore,

$$
\left.\frac{\partial f(x, z)}{\partial x}\right|_{x=1}=\frac{2 q z^{2}}{\left(1-q^{2}\right)(z-1)^{2}}
$$

which corresponds to the fact, that the mean of the total variation of a word with $n$ letters is $\frac{2 q}{1-q^{2}}(n-1)$. Also, we obtain

$$
\begin{aligned}
& \left.\frac{\partial^{2} f(x, z)}{\partial x^{2}}\right|_{x=1} \\
& =\frac{2 q z^{2}\left((1-q)\left(-q^{4}+q^{3}-4 q^{2}+q-1\right) z^{2}+\left(\left(q^{3}-5 q+3\right) q^{2}+1\right) z+2 q(q+1)\left(q^{2}+q+1\right)(1-q)\right)}{(1-q)^{3}(q+1)^{3}\left(q^{2}+q+1\right)(1-z)^{3}} .
\end{aligned}
$$

\section{Up-down words over $\mathbb{N}$ following the pattern $<\geq<\geq \cdots<\geq$}

\subsection{Up-down words of odd length}

The aim of this subsection is to find the occurrence probabilities of up-down words with odd length and given total variation. We note that recent work [2] of the present authors is dedicated to the $q$-enumeration of up-down words (following various patterns) by the number of rises.

Analogously to the previous section, we introduce the following generating functions. Let

$$
f(z, x)=\sum_{n \geq 0, r \geq 0} p(2 n+1, r) z^{2 n+1} x^{r}
$$

where $p(2 n+1, r)$ is the probability that a word of length $2 n+1$ has total variation $r$, and let

$$
F(z, x, t)=\sum_{n \geq 0, r \geq 0, i \geq 1} g(2 n+1, r, i) z^{2 n+1} x^{r} t^{i}=\sum_{n \geq 0} a_{2 n+1}(x, t) z^{2 n+1}
$$

where $g(2 n+1, r, i)$ is the occurrence probability of a word of length $2 n+1$, last letter $i\left(\omega_{2 n+1}=i\right)$ and total variation $r$. Again, $f(z, x)=F(z, x, 1)$.

We proceed, as in the previous section, inductively. Assume we have a random word of length $2 n+1$ with last letter $i, \omega=\omega_{1} \ldots \omega_{2 n} i$ and total variation $r$, with arbitrarily fixed $i \geq 1$ and $r>0$. Suppose we add two letters, $k$ and $j$, after the last letter $i$, and thus get a new word of length $2 n+3, \omega^{\prime}=\omega_{1} \ldots \omega_{2 n} i k j$. Then $i<k, k \geq j$ and the total variation of $\omega^{\prime}$ is $r+2 k-(i+j)$. This is reflected by the following expression

$$
\sum_{k>i} p q^{k-1} \sum_{j \leq k} p q^{j-1} \cdot t^{j} \cdot x^{2 k-(i+j)}
$$

This leads to the substitution

$$
t^{i} \longrightarrow \frac{p^{2} t}{(x-q t)} \cdot\left(\frac{1}{\left(1-q x^{2}\right)}\left(q x^{2}\right)^{i}-\frac{q t}{x\left(1-q^{2} x t\right)}\left(q^{2} x t\right)^{i}\right),
$$

or, equivalently,

$$
t^{i} \longrightarrow A(x, t)\left(\frac{1}{\left(1-q x^{2}\right)}\left(q x^{2}\right)^{i}-B(x, t) \cdot\left(q^{2} x t\right)^{i}\right)
$$


where $A(x, t)=\frac{p^{2} t}{(x-q t)}$ and $B(x, t)=\frac{q t}{x\left(1-q^{2} x t\right)}$.

Herefrom we obtain, for $n \geq 0$,

$$
a_{2 n+3}(x, t)=A(x, t)\left(\frac{1}{1-q x^{2}} a_{2 n+1}\left(x, q x^{2}\right)-B(x, t) \cdot a_{2 n+1}\left(x, q^{2} x t\right)\right)
$$

which, after multiplication by $z^{2 n+3}$ and then summing up for $n \geq 0$, leads to

$$
F(z, x, t)=a_{1}(x, t) z+A(x, t) z^{2}\left(\frac{1}{1-q x^{2}} F\left(z, x, q x^{2}\right)-B(x, t) \cdot F\left(z, x, q^{2} x t\right)\right),
$$

with $a_{1}(x, t)=\sum_{k \geq 1} p q^{k-1} t^{k}=\frac{p t}{1-q t}$. We solve the functional equation by iteration.

$$
\begin{aligned}
F(z, x, t)= & \frac{p t z}{1-q t}+A(x, t) z^{2}\left(\frac{1}{1-q x^{2}} F\left(z, x, q x^{2}\right)-B(x, t) \cdot[\right. \\
& \frac{p q^{2} x t z}{1-q^{3} x t}+A\left(x, q^{2} x t\right) z^{2}\left(\frac{1}{1-q x^{2}} F\left(z, x, q x^{2}\right)-B\left(x, q^{2} x t\right) \cdot[\right. \\
& \frac{p q^{4} x^{2} t z}{1-q^{5} x^{2} t}+A\left(x, q^{4} x^{2} t\right) z^{2}\left(\frac{1}{1-q x^{2}} F\left(z, x, q x^{2}\right)-B\left(x, q^{4} x^{2} t\right) \cdot[\right. \\
& \frac{p q^{6} x^{3} t z}{1-q^{7} x^{3} t}+A\left(x, q^{6} x^{3} t\right) z^{2}\left(\frac{1}{1-q x^{2}} F\left(z, x, q x^{2}\right)-B\left(x, q^{6} x^{3} t\right) \cdot[\right. \\
& \left.\left.\left.\left.\left.\left.\left.\left.\frac{p q^{8} x^{4} t z}{1-q^{9} x^{4} t}+\ldots\right]\right)\right]\right)\right]\right)\right]\right),
\end{aligned}
$$

and thus

$$
\begin{aligned}
F(z, x, t)= & \sum_{k \geq 1}\left(\frac{A\left(x, q^{2(k-1)} x^{k-1} t\right)}{1-q x^{2}} F\left(z, x, q x^{2}\right) z^{2}+\frac{p q^{2(k-1)} x^{k-1} t}{1-q^{2 k-1} x^{k-1} t} z\right) \\
& \times(-1)^{k-1} z^{2(k-1)} \prod_{i=1}^{k-1} A\left(x, q^{2(i-1)} x^{i-1} t\right) B\left(x, q^{2(i-1)} x^{i-1} t\right) .
\end{aligned}
$$

For $t=q x^{2}$ we get

$$
\begin{aligned}
F\left(z, x, q x^{2}\right)= & \sum_{k \geq 1}(-1)^{k-1} z^{2(k-1)} \prod_{i=1}^{k-1} A\left(x, q^{2 i-1} x^{i+1}\right) B\left(x, q^{2 i-1} x^{i+1}\right) \\
& \times\left[\frac{A\left(x, q^{2 k-1} x^{k+1}\right)}{1-q x^{2}} F\left(z, x, q x^{2}\right) z^{2}+\frac{p q^{2 k-1} x^{k+1}}{1-q^{2 k} x^{k+1}} z\right]
\end{aligned}
$$

which yields

$$
F\left(z, x, q x^{2}\right)=\left(\sum_{k \geq 1}(-1)^{k-1} \frac{p q^{2 k-1} x^{k+1}}{1-q^{2 k} x^{k+1}} z^{2 k-1} \prod_{i=1}^{k-1} A\left(x, q^{2 i-1} x^{i+1}\right) B\left(x, q^{2 i-1} x^{i+1}\right)\right) /
$$




$$
\left(1-\sum_{k \geq 1}(-1)^{k-1} \frac{A\left(x, q^{2 k-1} x^{k+1}\right)}{1-q x^{2}} z^{2 k} \prod_{i=1}^{k-1} A\left(x, q^{2 i-1} x^{i+1}\right) B\left(x, q^{2 i-1} x^{i+1}\right)\right) .
$$

Consequently,

$$
\begin{aligned}
& F(z, x, t)= \\
& \sum_{n \geq 1}\left(\frac{A\left(x, q^{2(n-1)} x^{n-1} t\right)}{1-q x^{2}} z^{2} \cdot \frac{\sum_{k \geq 1}(-1)^{k-1} \frac{p q^{2 k-1} x^{k+1}}{1-q^{2 k} x^{k+1}} z^{2 k-1} \prod_{i=1}^{k-1} A\left(x, q^{2 i-1} x^{i+1}\right) B\left(x, q^{2 i-1} x^{i+1}\right)}{1-\sum_{k \geq 1}(-1)^{k-1} \frac{A\left(x, q^{2 k-1} x^{k+1}\right)}{1-q x^{2}} z^{2 k} \prod_{i=1}^{k-1} A\left(x, q^{2 i-1} x^{i+1}\right) B\left(x, q^{2 i-1} x^{i+1}\right)}\right. \\
& \left.\quad+\frac{p q^{2(n-1)} x^{n-1} t}{1-q^{2 n-1} x^{n-1} t} z\right)(-1)^{(n-1)} z^{2(n-1)} \prod_{i=1}^{n-1} A\left(x, q^{2(i-1)} x^{i-1} t\right) B\left(x, q^{2(i-1)} x^{i-1} t\right) .
\end{aligned}
$$

We have

$$
\prod_{i=1}^{k-1} A\left(x, q^{2 i-1} x^{i+1}\right)=\prod_{i=1}^{k-1} \frac{p^{2} q^{2 i-1} x^{i+1}}{\left(x-q \cdot q^{2 i-1} x^{i+1}\right)}=\frac{p^{2(k-1)} q^{(k-1)^{2}} x^{\frac{k(k-1)}{2}}}{\left(q^{2} x ; q^{2} x\right)_{k-1}}
$$

and

$$
\prod_{i=1}^{k-1} B\left(x, q^{2 i-1} x^{i+1}\right)=\prod_{i=1}^{k-1} \frac{q \cdot q^{2 i-1} x^{i+1}}{x\left(1-q^{2} x \cdot q^{2 i-1} x^{i+1}\right)}=\frac{q^{k(k-1)} x^{\frac{k(k-1)}{2}}}{\left(q^{3} x^{3} ; q^{2} x\right)_{k-1}},
$$

and thus

$$
\prod_{i=1}^{k-1} A\left(x, q^{2 i-1} x^{i+1}\right) B\left(x, q^{2 i-1} x^{i+1}\right)=\frac{p^{2(k-1)} q^{(2 k-1)(k-1)} x^{k(k-1)}}{\left(q^{2} x ; q^{2} x\right)_{k-1}\left(q^{3} x^{3} ; q^{2} x\right)_{k-1}} .
$$

Moreover, since

$$
\prod_{i=1}^{n-1} A\left(x, q^{2(i-1)} x^{i-1} t\right)=\prod_{i=1}^{n-1} \frac{p^{2} q^{2(i-1)} x^{i-1} t}{\left(x-q \cdot q^{2(i-1)} x^{i-1} t\right)}=\frac{p^{2(n-1)} q^{(n-2)(n-1)} x^{\frac{(n-4)(n-1)}{2}} t^{n-1}}{\left(q \frac{t}{x} ; q^{2} x\right)_{n-1}}
$$

and

$$
\prod_{i=1}^{n-1} B\left(x, q^{2(i-1)} x^{i-1} t\right)=\prod_{i=1}^{n-1} \frac{q \cdot q^{2(i-1)} x^{i-1} t}{x\left(1-q^{2} x \cdot q^{2(i-1)} x^{i-1} t\right)}=\frac{q^{(n-1)^{2}} x^{\frac{(n-4)(n-1)}{2}} t^{n-1}}{\left(q^{2} x t ; q^{2} x\right)_{n-1}},
$$

we have

$$
\prod_{i=1}^{n-1} A\left(x, q^{2(i-1)} x^{i-1} t\right) B\left(x, q^{2(i-1)} x^{i-1} t\right)=\frac{p^{2(n-1)} q^{(2 n-3)(n-1)} x^{(n-4)(n-1)} t^{2(n-1)}}{\left(q_{x}^{t} ; q^{2} x\right)_{n-1}\left(q^{2} x t ; q^{2} x\right)_{n-1}} .
$$

These yield

$$
\begin{aligned}
& F(z, x, t)= \\
& \sum_{n \geq 1}(-1)^{n-1}\left(\frac{A\left(x, q^{2(n-1)} x^{(n-1)} t\right)}{1-q x^{2}} z^{2} \cdot \frac{\sum_{k \geq 1}(-1)^{k-1} \frac{p q^{2 k-1} x^{k+1}}{1-q^{2 k} x^{k+1}} \cdot \frac{p^{2(k-1)} q^{(2 k-1)(k-1)} x^{k(k-1)}}{\left.q^{2} x ; q^{2} x\right)_{k-1}\left(q^{3} x^{3} ; q^{2} x\right)_{k-1}} z^{2 k-1}}{1-\sum_{k \geq 1}(-1)^{k-1} \frac{A\left(x, q^{2 k-1} x^{k+1}\right)}{1-q x^{2}} \cdot \frac{p^{2(k-1)} q^{(2 k-1)(k-1)} x^{k(k-1)}}{\left(q^{2} x ; q^{2} x\right)_{k-1}\left(q^{3} x^{3} ; q^{2} x\right)_{k-1}}} z^{2 k}\right.
\end{aligned}
$$




$$
\begin{aligned}
& \left.+\frac{p q^{2(n-1)} x^{n-1} t}{1-q^{2 n-1} x^{n-1} t} z\right) z^{2(n-1)} \frac{p^{2(n-1)} q^{(2 n-3)(n-1)} x^{(n-4)(n-1)} t^{2(n-1)}}{\left(q \frac{t}{x} ; q^{2} x\right)_{n-1}\left(q^{2} x t ; q^{2} x\right)_{n-1}} \\
= & \sum_{n \geq 1}(-1)^{n-1}\left(\frac{p^{2} q^{2(n-1)} x^{n-1} t}{\left(1-q x^{2}\right)\left(x-q^{2 n-1} x^{n-1} t\right)} \cdot \frac{\sum_{k \geq 1} \frac{(-1)^{k-1} p^{2 k-1} q^{k(2 k-1)} x^{k^{2}+1}}{\left(1-q^{2 k} x^{k+1}\right)\left(q^{2} x ; q^{2} x\right)_{k-1}\left(q^{3} x^{3} ; q^{2} x\right)_{k-1}} z^{2 k+1}}{1-\sum_{k \geq 1} \frac{(-1)^{k-1} p^{2 k} q^{k(2 k-1)} x^{k^{2}}}{\left(1-q x^{2}\right)\left(1-q^{2 k} x^{k}\right)\left(q^{2} x ; q^{2} x\right)_{k-1}\left(q^{3} x^{3} ; q^{2} x\right)_{k-1}} z^{2 k}}\right. \\
& \left.+\frac{p q^{2(n-1)} x^{n-1} t}{1-q^{2 n-1} x^{n-1} t} z\right) \frac{p^{2(n-1)} q^{(2 n-3)(n-1)} x^{(n-4)(n-1)} t^{2(n-1)}}{\left(q \frac{t}{x} ; q^{2} x\right)_{n-1}\left(q^{2} x t ; q^{2} x\right)_{n-1}} \cdot z^{2(n-1)} .
\end{aligned}
$$

For $t=1$,

$$
\begin{aligned}
f(z, x)= & F(z, x, 1) \\
= & \sum_{n \geq 1}(-1)^{n-1}\left(\frac{p^{2} q^{2(n-1)} x^{n-1}}{\left(1-q x^{2}\right)\left(x-q^{2 n-1} x^{n-1}\right)} \cdot \frac{\sum_{k \geq 1} \frac{(-1)^{k-1} p^{2 k-1} q^{k(2 k-1)} x^{k^{2}+1}}{\left(1-q^{2 k} x^{k+1}\right)\left(q^{2} x ; q^{2} x\right)_{k-1}\left(q^{3} x^{3} ; q^{2} x\right)_{k-1}} z^{2 k+1}}{1-\sum_{k \geq 1} \frac{(-1)^{k-1} p^{2 k} q^{k(2 k-1)} x^{2}}{\left(1-q x^{2}\right)\left(1-q^{2 k} x^{k}\right)\left(q^{2} x ; q^{2} x\right)_{k-1}\left(q^{3} x^{3} ; q^{2} x\right)_{k-1}} z^{2 k}}\right. \\
& \left.+\frac{p q^{2(n-1)} x^{n-1}}{1-q^{2 n-1} x^{n-1}} z\right) \frac{p^{2(n-1)} q^{(2 n-3)(n-1)} x^{(n-4)(n-1)}}{\left(\frac{q}{x} ; q^{2} x\right)_{n-1}\left(q^{2} x ; q^{2} x\right)_{n-1}} \cdot z^{2(n-1)}
\end{aligned}
$$

Thus for $x=1$,

$$
\begin{aligned}
& f(z, 1)=F(z, 1,1) \\
& =\sum_{n \geq 1}(-1)^{n-1}\left(\frac{p q^{2(n-1)}}{1-q^{2 n-1}} \cdot \frac{\sum_{k \geq 1} \frac{(-1)^{k-1} p^{2 k-1} q^{k(2 k-1)}}{\left(1-q^{2 k}\right)\left(q^{2} ; q^{2}\right)_{k-1}\left(q^{3} ; q^{2}\right)_{k-1}} z^{2 k+1}}{1-\sum_{k \geq 1} \frac{(-1)^{k-1} p^{2 k} q^{k(2 k-1)}}{(1-q)\left(1-q^{2 k}\right)\left(q^{2} ; q^{2}\right)_{k-1}\left(q^{3} ; q^{2}\right)_{k-1}} z^{2 k}}+\frac{p q^{2(n-1)}}{1-q^{2 n-1}} z\right) \\
& \cdot \frac{p^{2(n-1)} q^{(2 n-3)(n-1)}}{\left(q ; q^{2}\right)_{n-1}\left(q^{2} ; q^{2}\right)_{n-1}} \cdot z^{2(n-1)} \\
& =\sum_{n \geq 1} \frac{(-1)^{n-1} p^{2 n-1} q^{(2 n-1)(n-1)}}{\left(q ; q^{2}\right)_{n}\left(q^{2} ; q^{2}\right)_{n-1}\left(1-\sum_{k \geq 1} \frac{(-1)^{k-1} p^{2 k-1} q^{k(2 k-1)}}{\left(1-q^{2 k}\right)\left(q^{2} ; q^{2}\right)_{k-1}\left(q^{3} ; q^{2}\right)_{k-1}} z^{2 k}\right)} z^{2 n-1} \\
& =\frac{\sum_{k \geq 1} \frac{(-1)^{k-1} p^{2 k-1} q^{(2 k-1)(k-1)} z^{2 k-1}}{\left(q ; q^{2}\right)_{k}\left(q^{2} ; q^{2}\right)_{k-1}}}{1-\sum_{k \geq 1} \frac{(-1)^{k-1} p^{2 k} q^{k(2 k-1)} z^{2 k}}{\left(q ; q^{2}\right)_{k}\left(q^{2} ; q^{2}\right)_{k}}}=\frac{\sum_{n \geq 0} \frac{(-1)^{n} p^{2 n+1} q^{(2 n+1) n} z^{2 n+1}}{(q ; q)_{2 n+1}}}{\sum_{n \geq 0} \frac{(-1)^{n} p^{2 n} q^{(2 n-1) n} z^{2 n}}{(q ; q)_{2 n}}},
\end{aligned}
$$

which corresponds to the case when neither the last letter nor the total variation of the words are considered.

\subsection{Up-down words of even length}

In this subsection we are looking for the probabilities of occurrence of up-down words with even length and given total variation.

We proceed inductively.

Assume we have a random word of length $2 n+2, n \geq 0$, with last letter $l, \omega=\omega_{1} \ldots \omega_{2 n+1} l$, with arbitrarily fixed $l \geq 1$ and $r>0$. This can be obtained by adding the letter $l$, after the last letter $j$ of the 
random up-down word $\omega^{\prime}=\omega_{1} \ldots \omega_{2 n+1}$. Then $l>j$, and the total variation of $\omega$ is $r=r^{\prime}+l-j$, where $r^{\prime}$ denotes the total variation of $\omega^{\prime}$. This is reflected by the following expression

$$
\left(\sum_{k>i} p q^{k-1} \sum_{j \leq k} p q^{j-1} \cdot t^{j} \cdot x^{2 k-(i+j)}\right) \sum_{l>j} p q^{l-1} z x^{l-j} t^{l} .
$$

We introduce the generating function

$$
g(z, x):=\sum_{n \geq 0, r \geq 0} p(2 n+2, r) z^{2 n+2} x^{r},
$$

where $p(2 n+2, r)$ is the probability that an up-down word of length $2 n+2$ has total variation $r$. Since $\sum_{l>j} p q^{l-1} z x^{l-j} t^{l}=\frac{p z x t}{1-q x t}(q t)^{j}$, one can obtain the sum (19) by replacing $t^{j}$ in the sum (13) by $\frac{p z x t}{1-q x t}(q t)^{j}$. Therefore, for $t=1$ the role played by $F(z, x, 1)$ in the case of up-down words of odd length is played here by $\frac{p z x}{1-q x} F(z, x, q)$. We then have

$$
g(z, x)=\frac{p z x}{1-q x} F(z, x, q)
$$

i.e.,

$$
\begin{aligned}
g(z, x)= & \frac{p x}{1-q x} \sum_{n \geq 1}\left(\frac{p^{2} q^{2 n-1} x^{n-1}}{\left(1-q x^{2}\right)\left(x-q^{2 n} x^{n-1}\right)} \cdot \frac{\sum_{k \geq 1} \frac{(-1)^{k-1} p^{2 k-1} q^{k(2 k-1)} x^{2}+1}{\left(1-q^{2 k} x^{k+1}\right)\left(q^{2} x ; q^{2} x\right)_{k-1}\left(q^{3} x^{3} ; q^{2} x\right)_{k-1}} z^{2 k+1}}{1-\sum_{k \geq 1} \frac{(-1)^{k-1} p^{2 k} q^{k(2 k-1)} x^{k^{2}}}{\left(1-q x^{2}\right)\left(1-q^{2 k} x^{k}\right)\left(q^{2} x ; q^{2} x\right)_{k-1}\left(q^{3} x^{3} ; q^{2} x\right)_{k-1}} z^{2 k}}\right. \\
& \left.+\frac{p q^{2 n-1} x^{n-1}}{1-q^{2 n} x^{n-1}} z\right) \frac{(-1)^{n-1} p^{2(n-1)} q^{(2 n-1)(n-1)} x^{(n-4)(n-1)}}{\left(\frac{q^{2}}{x} ; q^{2} x\right)_{n-1}\left(q^{3} x ; q^{2} x\right)_{n-1}} \cdot z^{2 n-1} .
\end{aligned}
$$

\section{Carlitz words}

Carlitz words are words over the alphabet $\mathbb{N}$ with the property that any two adjacent letters are distinct. In this section we study the occurrence probabilities of Carlitz words with given total variation. Carlitz compositions and random Carlitz compositions of natural numbers were studied by several authors, e.g., Carlitz [1], Knopfmacher and Prodinger [4], Goh and Hitczenko [3], Louchard and Prodinger [5]. We use the notations from Section 2, namely

$$
f(z, x)=\sum_{n \geq 1, r \geq 0} p(n, r) z^{n} x^{r}
$$

where $p(n, r)$ is in this case the probability that a Carlitz word of length $n$ has total variation $r$, and

$$
F(z, x, t)=\sum_{n \geq 1, r \geq 0, i \geq 1} g(n, r, i) z^{n} x^{r} t^{i},
$$

where $g(n, r, i)$ is the occurrence probability of a Carlitz word of length $n$, last letter $i\left(\omega_{n}=i\right)$ and total variation $r$. We have $f(z, x)=F(z, x, 1)$. 
Assume we have a random Carlitz word of length $n$ with last letter $i, \omega=\omega_{1} \ldots \omega_{n-1} i$, which has total variation $r$, with arbitrarily fixed $i \geq 1$ and $r \geq 0$. Suppose we add a letter, $j$, after the last letter $i$, and thus get a new word of length $n+1, \omega^{\prime}=\omega_{1} \ldots \omega_{n-1} i j$. Then either $j>i$ and the new word $\omega^{\prime}$ has total variation $r+j-i$, or $j<i$ and then $\omega^{\prime}$ has total variation $r+i-j$.

This is reflected by the following expression

$$
\sum_{j=1}^{i-1} p q^{j-1} \cdot x^{i-j} \cdot t^{j}+\sum_{j \geq i+1} p q^{j-1} \cdot x^{j-i} \cdot t^{j}
$$

that leads to the substitution

$$
t^{i} \longrightarrow \frac{p t}{(x-q t)} x^{i}+\left(\frac{p t x}{1-q t x}-\frac{p x}{q(x-q t)}\right)(q t)^{i} .
$$

Let again $F(z, x, t)=\sum_{n \geq 1} a_{n}(x, t) z^{n}$. For $n \geq 1$, we get from 24,

$$
a_{n+1}(x, t)=\frac{p t}{(x-q t)} a_{n}(x, x)+\left(\frac{p x t}{1-q t x}-\frac{p}{q(x-q t)}\right) a_{n}(x, q t)
$$

which, after multiplication by $z^{n+1}$ and then summming up for $n \geq 1$, yields

$$
F(z, x, t)=a_{1}(x, t) z+\frac{p t}{(x-q t)} z F(z, x, x)+\left(\frac{p x t}{1-q t x}-\frac{p}{q(x-q t)}\right) z F(z, x, q t),
$$

where $a_{1}(x, t)=\sum_{k \geq 1} p q^{k-1} t^{k}=\frac{p t}{1-q t}$.

We introduce the notation

$$
T(x, t):=\frac{p t x}{1-q t x}-\frac{p}{q(x-q t)}
$$

and obtain from the functional equation 26,

$$
\begin{aligned}
F(z, x, t)= & \frac{p t}{1-q t} z+\frac{p t}{(x-q t)} z F(z, x, x)+T(x, t) z[ \\
& \frac{p q t}{1-q^{2} t} z+\frac{p q t}{\left(x-q^{2} t\right)} z F(z, x, x)+T(x, q t) z[ \\
& \frac{p q^{2} t}{1-q^{3} t} z+\frac{p q^{2} t}{\left(x-q^{3} t\right)} z F(z, x, x)+T\left(x, q^{2} t\right) z[ \\
& \frac{p q^{3} t}{1-q^{4} t} z+\frac{p q^{3} t}{\left(x-q^{4} t\right)} z F(z, x, x)+T\left(x, q^{3} t\right) z[ \\
& \left.\left.\left.\left.\frac{p q^{4} t}{1-q^{5} t} z+\ldots\right]\right]\right]\right] .
\end{aligned}
$$

This leads to

$$
F(z, x, t)=\sum_{k \geq 0} p^{k+1} t^{k+1} z^{k+1} q^{\frac{k(k+1)}{2}} \prod_{l=1}^{k} T\left(x, q^{l-1} t\right)\left(\frac{1}{1-q^{k+1} t}+\frac{1}{x-q^{k+1} t} F(z, x, x)\right) .
$$


For $t=x$, we get

$$
F(z, x, x)=\sum_{k \geq 0} p^{k+1} x^{k+1} z^{k+1} q^{\frac{k(k+1)}{2}} \prod_{l=1}^{k} T\left(x, q^{l-1} x\right)\left(\frac{1}{1-q^{k+1} x}+\frac{1}{x-q^{k+1} x} F(z, x, x)\right)
$$

and thus

$$
F(z, x, x)=\frac{\sum_{k \geq 0} p^{k+1} x^{k+1} z^{k+1} q^{\frac{k(k+1)}{2}} \cdot \frac{1}{1-q^{k+1} x} \prod_{l=1}^{k} T\left(x, q^{l-1} x\right)}{1-\sum_{k \geq 0} p^{k+1} x^{k} z^{k+1} q^{\frac{k(k+1)}{2}} \cdot \frac{1}{1-q^{k+1}} \prod_{l=1}^{k} T\left(x, q^{l-1} x\right)} .
$$

This yields

$$
\begin{aligned}
F(z, x, t)= & \sum_{n \geq 0} p^{n+1} t^{n+1} z^{n+1} q^{\frac{n(n+1)}{2}} \prod_{j=1}^{n} T\left(x, q^{j-1} t\right) \\
& \times\left(\frac{1}{1-q^{n+1} t}+\frac{1}{x-q^{n+1} t} \frac{\sum_{k \geq 0} p^{k+1} x^{k+1} z^{k+1} q^{\frac{k(k+1)}{2}} \prod_{l=1}^{k} T\left(x, q^{l-1} x\right) \frac{1}{1-q^{k+1} x}}{1-\sum_{k \geq 0} p^{k+1} x^{k+1} z^{k+1} q^{\frac{k(k+1)}{2}} \prod_{l=1}^{k} T\left(x, q^{l-1} x\right) \frac{1}{x-q^{k+1} x}}\right)
\end{aligned}
$$

and, for $t=1$,

$$
\begin{aligned}
f(z, x)= & F(z, x, 1)=\sum_{n \geq 0} p^{n+1} z^{n+1} q^{\frac{n(n+1)}{2}} \prod_{j=1}^{n} T\left(x, q^{j-1}\right)\left(\frac{1}{1-q^{n+1}}\right. \\
& \left.+\frac{1}{x-q^{n+1}} \cdot \frac{\sum_{k \geq 0} p^{k+1} x^{k+1} z^{k+1} q^{\frac{k(k+1)}{2}} \prod_{l=1}^{k} T\left(x, q^{l-1} x\right) \frac{1}{1-q^{k+1} x}}{1-\sum_{k \geq 0} p^{k+1} x^{k+1} z^{k+1} q^{\frac{k(k+1)}{2}} \prod_{l=1}^{k} T\left(x, q^{l-1} x\right) \frac{1}{x-q^{k+1} x}}\right) .
\end{aligned}
$$

Since $T\left(1, q^{j-1}\right)=-\frac{p}{q}$, we obtain for $x=1$,

$$
\begin{aligned}
f(z, 1)= & F(z, 1,1)=\sum_{n \geq 0} p^{n+1} z^{n+1} q^{\frac{n(n+1)}{2}} \prod_{j=1}^{n} T\left(1, q^{j-1}\right)\left(\frac{1}{1-q^{n+1}}\right. \\
& \left.+\frac{1}{1-q^{n+1}} \cdot \frac{\sum_{k \geq 0} p^{k+1} z^{k+1} q^{\frac{k(k+1)}{2}} \prod_{l=1}^{k} T\left(1, q^{l-1} x\right) \frac{1}{1-q^{k+1}}}{1-\sum_{k \geq 0} p^{k+1} z^{k+1} q^{\frac{k(k+1)}{2}} \prod_{l=1}^{k} T\left(1, q^{l-1}\right) \frac{1}{1-q^{k+1}}}\right) \\
= & \sum_{n \geq 0}(-1)^{n} p^{2 n+1} z^{n+1} q^{\frac{n(n-1)}{2}} \cdot \frac{1}{1-q^{n+1}} \\
& \times \frac{1}{1-\sum_{k \geq 0}(-1)^{k} p^{2 k+1} z^{k+1} q^{\frac{k(k-1)}{2}} \cdot \frac{1}{1-q^{k+1}}} \\
= & -1+1 /\left(1-\sum_{n \geq 0}(-1)^{n} p^{2 n+1} q^{\frac{n(n-1)}{2}} \cdot \frac{z^{n+1}}{1-q^{n+1}}\right) .
\end{aligned}
$$

This corresponds to the case when neither the last letter nor the total variation of the words are considered. 


\section{References}

[1] L. Carlitz, Restricted compositions, The Fibonacci Quarterly, Vol. 14, 1976, 254-264.

[2] L.L. Cristea and H. Prodinger, q-enumeration of up-down words by number of rises, The Fibonacci Quarterly 46/47 (2008/2009), no. 2, 126-134.

[3] W.M.Y. Goh and P. Hitczenko, Average Number of Distinct Path Sizes in a Random Carlitz Composition, European Journal of Combinatorics, 2002, 23, 647-657.

[4] A. Knopfmacher and H. Prodinger, On Carlitz compositions, European Journal of Combinatorics, 19 , 1998, 579-589.

[5] G. Louchard and H. Prodinger, Probabilistic Analysis of Carlitz Compositions, Discrete Mathematics and Theoretical Computer Science, 5, 2002, 71-96.

[6] T. Mansour, Enumeration of words by the sum of differences between adjacent letters, Discrete Mathematics and Theoretical Computer Science, Vol. 11:1, 2009, 173-186. 